\title{
Numerical Fracture analysis of prestressed concrete beams
}

\author{
Timon Rabczuk ${ }^{1)}$ and Goangseup $\mathrm{Zi}^{2)}$
}

(Received May 29, 2008, Revised October 30, 2008, Accepted November 30, 2008)

\begin{abstract}
Fracture of prestressed concrete beams is studied with a novel and robust three-dimensional meshfree method. The meshfree method describes the crack as a set of cohesive crack segments and avoids the representation of the crack surface. It is ideally suited for a large number of cracks. The crack is modeled by splitting particles into two particles on opposite sides of the crack segment and the shape functions of neighboring particles are modified in a way the discontinuous displacement field is captured appropriately. A simple, robust and efficient way to determine, on which side adjacent particles of the corresponding crack segment lies, is proposed. We will show that the method does not show any "mesh" orientation bias and captures complicated failure patterns of experimental data well.
\end{abstract}

Keywords: reinforced concrete, meshfree methods, fracture, cohesive models.

\section{Introduction}

The simulation of large sets of evolving cracks in concrete structure still poses substantial difficulties on numerical methods. Modelling of discrete cracks is essential for reliability and safety analysis of reinforced concrete structures. Crack models in numerical methods can be classified into two categories:

- Methods that enforce crack path continuity and

- Methods that do not enforce crack path continuity

The first category can be classified into two sub-categories, i.e. methods that can handle embedded cracks and methods that cannot have embedded cracks. Inter-element separation methods (Xu and Needleman; ${ }^{29,30}$ Camacho and Ortiz; ${ }^{10}$ Ortiz et al.; ${ }^{20}$ Zhou and Molinari) ${ }^{31}$ belong to the latter group. In inter-element separation models, cracks are only allowed to develop along existing inter-element edges. This endows the method with comparative simplicity, but can result in an overestimate of the fracture energy when the actual crack paths are not coincident with element edges. The results severely depend on the shape and mesh orientation of the mesh. This mesh dependence can only be alleviated by excessive remeshing that is computationally expensive. Methods that are capable to embed the discontinuity, i.e. the crack, in the element are embedded elements (Belytschko et al.; ${ }^{3}$ Oliver; ${ }^{16}$ Oliver et al.; ${ }^{17,18}$ Armero and Garikipati) ${ }^{1}$ and the extended finite element method (XFEM) (Belytschko and Black; ${ }^{2}$ Moes et al.; ${ }^{15}$ Belytschko et al.; ${ }^{7}$ Bordas et al.). ${ }^{32-35}$ In embedded elements, the crack can propagate only one element at a time and the cracks open piecewise constant. In the extended finite element method, the crack can open linearly (in case of linear shape functions) and non-

\footnotetext{
${ }^{1)}$ University of Canterbury, Dept. of Mechanical Engineering, Christchurch, New Zealand. E-mail: timon.rabczuk@canterbury.ac.nz

${ }^{2)}$ KCI Member, Korea University, Department of Civil \& Environmental Engineering, Seoul, Korea.

Copyright (c) 2008, Korea Concrete Institute. All rights reserved, including the making of copies without the written permission of the copyright proprietors.
}

linear (in case of higher order shape functions). Both methods require crack path continuity. The crack is usually described by level sets though this is not mandatory. When many cracks and when bifurcating and joining cracks occur, methods that enforce crack path continuity become cumbersome. Tracing the crack is one of the most difficult tasks in these methods, especially in 3D. The situation in meshfree methods is similar. Most meshfree methods consider the crack as continuous surface, Krysl and Belytschko; ${ }^{13}$ Belytschko et al.; ${ }^{8}$ Belytschko and Lu; ${ }^{5}$ Belytschko et al.; ${ }^{6}$ Belytschko and Tabbara. ${ }^{9}$ Hence, the difficulties in meshfree methods are similar as compared to XFEM though no special treatment for "branched elements" have to be considered due to the "meshfree" character of the discretization.

Recently, methods have been proposed that do not enforce crack path continuity but that are capable of capturing the jump in the displacement field typical for strong discontinuity approaches, Remmers et al.; ${ }^{28}$ Rabczuk and Belytschko. ${ }^{23}$ Though these methods are less accurate than methods that enforce crack path continuity, they are easier to implement and usually better suited for problems with excessive cracking since they do not require algorithms to track the crack paths. There is also no need of representing the crack as continuous surface. This paper is motivated by the cracking particle method $^{23}$ where the crack was modelled by a discontinuous enrichment that can be arbitrary aligned in the body at each particle (or node). The model of a continuous crack then consists of a set of contiguous cracked particles. In, ${ }^{26}$ it was shown that a discontinuous enrichment of cracked particles is not sufficient and particles in the blending domain were excluded in the approximation of the discontinuous displacement field. Within this paper, we follow the idea of modelling the crack as set of cracked particles. However, in contrast to the cracking particle method, we don't introduce additional unknowns in the variational formulation to capture the displacement discontinuity. Instead, the particles, where cracking is detected, are split into two particles lying on opposite sides of the crack. To capture the jump in the displacement field, the shape functions of the cracked particles are cut across the crack 
boundary similar to the visibility method, Belytschko et al.; ${ }^{8,6}$ Organ et al. ${ }^{19}$ Also the shape functions adjacent to the cracked particles are cut based on a simple condition that requires only the normal to the crack segment. The major advantage of the proposed method compared to the approach in Rabczuk and Belytschko $^{23}$ is that no additional unknowns need to be introduced. The method will be applied to prestressed concrete beams where a large number of cracks occurs.

We have focused on a meshfree method since it has certain advantages over finite elements in the context of fracture of reinforced concrete structures. First, a large number of cracks can be better dealt with methods that do not enforce crack path continuity and meshfree methods are better suited for this type of methods. Second, meshfree methods do not show a mesh orientation bias due to the isotropic (spherical) domain of influence. Third, h-adaptivity (useful for computational efficiency and increase of accuracy around the crack tip) can be incorporated easier in a meshfree method due to the absence of a mesh. Finally, the higher order continuity of meshfree methods give more accurate results in the stress and strain field that is needed to determine the direction of crack growth.

The article is arranged as follows: The governing equations are given in the next section. Then, we explain the new cracking concept and a simple procedure to determine on which side a particle is lying with respect to several crack segments. The discrete equations, the constitutive models and the cracking criterion are discussed afterwards. Finally, we apply the method to prestressed reinforced concrete beams under four-point-bending and show good agreement between experimental and numerical results.

\subsection{Governing equations}

The governing equation is the equation of equilibrium given by

$\nabla \cdot \boldsymbol{P}-\boldsymbol{b}=0 \forall \boldsymbol{X} \in \Omega \backslash \Gamma_{c}$

where $\boldsymbol{P}$ is the nominal stress tensor, $\boldsymbol{b}$ are the body forces, $\boldsymbol{X}$ are the material coordinates, $\nabla$ is the gradient operator with respect to the material coordinates and $\Gamma_{c}$ is the crack surface. The boundary conditions are

$$
\begin{aligned}
& \mathbf{u}(\mathbf{X})=\overline{\mathbf{u}}(\mathbf{X}) \text { on } \Gamma_{u} \\
& \mathbf{n} \cdot \mathbf{P}(\mathbf{X})=\overline{\mathbf{t}}(\mathbf{X}) \text { on } \Gamma_{t} \\
& \mathbf{n} \cdot \mathbf{P}^{-}=\mathbf{n} \cdot \mathbf{P}^{+}=\mathbf{t}_{c} \text { on } \Gamma_{t}
\end{aligned}
$$

where $\overline{\mathbf{u}}$ and $\overline{\mathbf{t}}$ are the prescribed displacements and tractions, respectively, $\mathbf{t}_{c}$ are the cohesive forces across the crack and $\boldsymbol{n}$ is the outward normal to the domain.

\subsection{Displacement field approximation and element- free galerkin (EFG) method}

The approximation of the displacement field in a Lagrangian description using the EFG method is given by

$$
\mathbf{u}(\mathbf{X})=p^{\mathbf{T}}(\mathbf{X}) \boldsymbol{a}(\mathbf{X})
$$

where $\boldsymbol{a}$ are the unknowns and $\boldsymbol{p}$ is a polynomial basis that we have chosen as $\mathbf{p}(\mathbf{X})=\left[\begin{array}{lll}1 & X & Y\end{array}\right]$. Minimizing the discrete weighted $L_{2}$ error norm

$$
J=\sum_{I \in S} w\left(\mathbf{X}-\mathbf{Y}_{1}, h\right)\left(\mathbf{p}_{I}^{T}(\mathbf{X}) \mathbf{a}(\mathbf{X})-\mathbf{u}_{I}\right)^{2}
$$

with respect to $\boldsymbol{a}$ leads to the approximation

$$
\mathbf{u}^{h}(\mathbf{X})=\sum_{I \in S} \Phi_{I}(\mathbf{X}) \mathbf{u}_{I}
$$

where $\Phi_{I}(\mathbf{X})$ is the shape function of particle $I, S$ is the set of neighbour particles for $\boldsymbol{X}, \mathbf{u}_{I}$ is the value at the particle at position $\mathbf{X}_{I}, \mathrm{w}\left(\mathbf{X}-\mathbf{X}_{I}, h\right)$ is a window or kernel function and $h$ is the interpolation radius of the window function. In the EFG method (see e.g. Belytschko and Lu; ${ }^{5}$ Belytschko et al.; Belytschko and Tabbara ${ }^{9}$ ), the shape functions can be derived from the minimization procedure as follows:

$$
\begin{aligned}
\Phi_{I}(\mathbf{X}) & =\mathbf{p}^{T}(\mathbf{X}) \cdot \mathbf{A}^{-1}(\mathbf{X}) \cdot \mathbf{B}(\mathbf{X}) \\
\mathbf{A}(\mathbf{X}) & =\sum_{J \in S} \mathbf{p}_{J}(\mathbf{X}) \mathbf{p}_{J}^{T}(\mathbf{X}) w\left(\mathbf{X}-\mathbf{X}_{J}, h\right) \\
\mathbf{B}(\mathbf{X}) & =\sum_{J \in S} \mathbf{p}_{J}(\mathbf{X}) w\left(\mathbf{X}-\mathbf{X}_{J}, h\right)
\end{aligned}
$$

Equations (5) and (6) are the EFG displacement approximation for a continuous displacement field. Now, let us consider a displacement field in the presence of a crack. The crack introduces a jump in the displacement field. This requires the modification of equations (5) and (6) in the vicinity of the crack. The basic idea is the same as in Rabczuk and Belytschko ${ }^{23}$ where the crack is modeled by a set of cracked particles as shown in Figure 1, simplified for the two-dimensional case. However, in contrast to, ${ }^{23}$ the displacement discontinuity is not introduced by additional unknowns in the variational formulation. Instead, a cracked particle is split into two particles lying on opposite sides of the crack segment as shown in Fig. 2. The discrete crack segments are restricted to lie between the two cracked particles. Crack opening is assumed to be piece-wise constant. Since the crack geometry is described by the set of cracked particles, we do not have to provide a representation for the geometry of the crack that entails additional complexity. At crack initiation, both particles will be placed at the same location but they separate during the course of the simulation. The crack segment is assumed to cross the entire domain of influence of the associated split cracked particle. For neighboring particles, the shape function is cut similar as in the visibility method, Belytschko et al.; ${ }^{6}$ Organ et al. ${ }^{19}$ Since the crack is not described continuously, the visibility criterion has to be modified. Here, we take advantage of the signed distance function with respect to the corresponding crack segment. More details are outlined below.

As previously mentioned, it becomes also necessary to "cut" the shape functions of particles adjacent to the cracked particles in order to approximate the jump in the displacement field. The approximation of the displacement field accounting for the jump in the displacement field can then be expressed as 

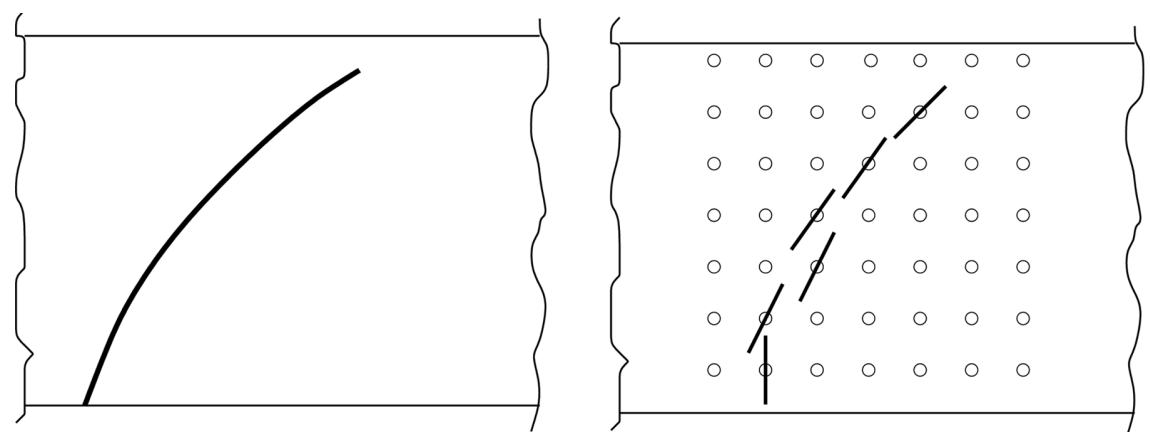

Fig. 1 Schematic on the right shows a crack model for the crack on the left.
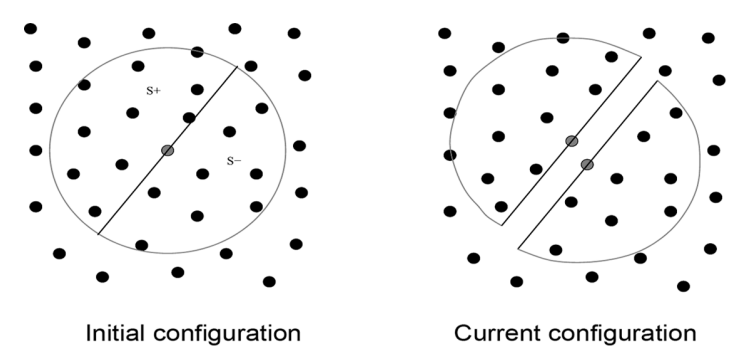

Fig. 2 Schematic on the right shows a crack model for the crack on the left.

$$
\mathbf{u}^{h}(\mathbf{X})=\sum_{I \in S} \Phi_{I}(\mathbf{X}) H\left(f_{I}(\mathbf{X})\right) \mathbf{u}_{I}
$$

where $H\left(f_{I}(\mathbf{X})\right)$ is the Heaviside function that is one on one side of the crack and zero on the other side of the crack and $f_{I}(\mathbf{X})$ is the signed distance function with respect to the corresponding cracked particle given by

$$
f_{I}(\mathbf{X})=\mathbf{n} \cdot\left(\mathbf{X}-\mathbf{X}_{I}\right)
$$

where $\boldsymbol{n}$ is the normal to the crack that is obtained from the cracking criterion discussed later. For a better illustration, consider Fig. 2 (right side). The red particles are cracked and are split into two new nodes at crack initiation. Note that at crack initiation, both particles coincide. Figure 2 shows exemplarily the representation of a crack for a two dimensional crack branching. Particle 1 lies in front of the "crack tip" and is outside of the domain of influence of all cracked particles and hence not influenced. For the green particle, the particles included in its domain of influence-shown by the green circle- is shown in blue colour. All other particles are lying on the opposite side with respect to the corresponding cracked particles and hence $H=0$. The "visibility check" of "uncracked" particles is done with respect to each (corresponding) crack segment in order to assemble the connectivities. Note that the only requirement is the initial position vector of the crack segment and the normal of the crack segment.

\subsection{Weak form and discretization}

The weak form of the equilibrium including a cohesive crack is given by: Find $\mathbf{u} \in V$ such that $\forall \delta \mathbf{u} \in V_{0}$

$$
\int_{\Omega}(\nabla \otimes \delta \mathbf{u})^{T}: P d \Omega-\int_{\Omega} \delta \mathbf{u} \cdot \mathrm{b} d \Omega-\int_{r_{t}} \delta \mathbf{u} \cdot \bar{t} d \Gamma-\int_{\Gamma_{c}} \delta[[\mathbf{u}]] \cdot \mathbf{t}_{c} d \Gamma=0
$$

where $\delta$ denotes the variation and where the trial functions $\boldsymbol{u}$ and the test functions $\delta \mathbf{u}$ lie in the following approximation spaces

$$
\begin{aligned}
& V=\left\{\mathbf{u}\left|\mathbf{u} \in H^{1}\right| \Gamma_{c}, \mathbf{u} \text { discontinuous on } \Gamma_{c}, \mathbf{u}=\mathbf{u} \text { on } \Gamma_{u}\right\} \\
& V=\left\{\delta \mathbf{u}\left|\delta \mathbf{u} \in H^{1}\right| \Gamma_{c}, \delta \mathbf{u} \text { discontinuous on } \Gamma_{c}, \delta \mathbf{u}=0 \text { on } \Gamma_{u}\right\}
\end{aligned}
$$

with $\Gamma=\Gamma_{c} \mathrm{Y} \Gamma_{t} \mathrm{Y} \Gamma_{u}$ and $\Gamma_{c} \mathrm{Y} \Gamma_{t} \mathrm{Y} \Gamma_{u}=0$ where $\Gamma_{c}$ is the crack surface. The [[ ]]-brackets in equation (9) denote the jump operator (in the displacement field). Substituting the approximation for the trial and test functions (the test functions have the same structure as the trial functions) into the variational formulation, equation (9), and performing a linearization, we obtain the final system of equations:

$$
\mathbf{K} \Delta \mathbf{u}=\mathbf{f}^{e x t}-\mathbf{f}^{\text {int }}-\mathbf{f}^{c o h}
$$

Where $\boldsymbol{K}$ is the tangential stiffness matrix of the system, $\Delta \mathbf{u}$ is the increment of the displacement vector and $\mathbf{f}^{\text {ext }}, \mathbf{f}^{\text {int }}$ and $\mathbf{f}^{\text {coh }}$ are the external force vector, the internal force vector and the cohesive force vector, respectively. We deal with non-linearities with the Newton-Raphson technique.

\subsection{Cracking criterion and cohesive/constitutive model}

Before cracking, we employed a continuum model outlined in [23]. A crack is initiated when the material loses stability. It can be shown that loss of material stability occurs when the minimum eigenvalue of the acoustic tensor $\boldsymbol{Q}$ is smaller than zero: $\min$ $\operatorname{eig}(\boldsymbol{Q})<0$ with

$$
\mathbf{Q}=\mathbf{n} \cdot \mathbf{A} \cdot \mathbf{n}
$$

with $\mathbf{A}=\mathbf{C}^{t}+\boldsymbol{\sigma} \otimes \delta$ where $\mathbf{C}^{t}$ is the consistent material tangen- 
tial stiffness matrix, $\sigma$ is the Cauchy stress tensor and $\delta$ is the Kronecker-delta. When the material loses material stability, $\boldsymbol{n}$ completely determines the orientation of the crack, i.e. $\boldsymbol{n}$ becomes the normal to the crack. The eigenvector $\boldsymbol{h}$ to the corresponding eigenvalue of $\boldsymbol{Q}$ at cracking determines the direction of the localization. For a pure mode I-crack $\boldsymbol{n}$ will be perpendicular to $\boldsymbol{h}$ while for pure mode II-crack $\boldsymbol{n}$ is parallel to $\boldsymbol{h}$. For mixed mode failure, the direction of $\boldsymbol{h}$ with respect to $\boldsymbol{n}$ will be somehow between the two extreme cases. Note that the bifurcation analysis gives generally several possible crack orientations. A criterion that reliably gave the correct orientation is to project the displacement gradient in localization direction and maximizing the following condition:

$$
g=\underbrace{\max }_{l}\left(n_{l} \cdot\left[\nabla \mathbf{u} \cdot \mathbf{h}_{I}\right]\right)
$$

where $l$ are the minima obtained from the material stability analysis.

A cohesive model is applied at the crack boundaries to account for the dissipated energy during crack opening. The cohesive law is of the form:

$$
t_{n}=\underbrace{\frac{t_{\max }}{\max }\left[\left[u_{n}\right]\right]}_{\text {history }} \exp (-\frac{t_{\max }}{G_{f}} \underbrace{\max }_{\text {history }}\left[\left[u_{n}\right]\right])\left[\left[u_{n}\right]\right]
$$

where the subscript $n$ indicates the normal component and the jump in the displacement field is given by

$$
\left[\left[u_{n}\right]\right]=\mathbf{n} \cdot[[\mathbf{u}]]
$$

with

$$
[[\mathbf{u}]]=\mathbf{u}^{+}-\mathbf{u}^{-}
$$

More details on the cohesive law can be found in [22].

\section{Results and discussion}

We consider three prestressed concrete beams without stirrup reinforcement. Details on the experimental set-up can be found in [36]. The first beam-beam I- has a rectangular cross section. The test setup and the dimensions of the beam are illustrated in Fig.3. The beam was prestressed with two tension wires (St 1470/1670) of $7 \mathrm{~mm}$ diameter prior to loading. The lower reinforcement was prestressed with a force of $26.25 \mathrm{kN}$, the upper one with a force of $11.25 \mathrm{kN}$. The beam failed in bending because of the plastic flow of the lower reinforcement followed by a failure of the concrete compression zone. Fig. 4 shows a cutout of the beam after the experiment. Crack number 2 and 3 are the cracks that caused the failure.

The test setup for the second beam-beam II- is shown in figure 5. In contrast to the first beam, the second beam has a I-cross section. The beam has two tension wires (St 1420/1570) of $12 \mathrm{~mm}$ diameter at the lower flange that were prestressed each with a force of $80 \mathrm{kN}$. The upper reinforcement (BSt 500) was not prestressed and had a diameter of $10 \mathrm{~mm}$. It was only installed due to transportation purposes. The beam failed suddenly because of a combined shear/pullout failure as illustrated in Fig. 6 (LHS). Crack number 6 caused the failure.

The third beam-beam III- has also a I-shaped cross section but the dimensions differ from beam II, see Fig. 7. The beam is prestressed with two tension wires (St 1420/1570) of $12 \mathrm{~mm}$ diameter

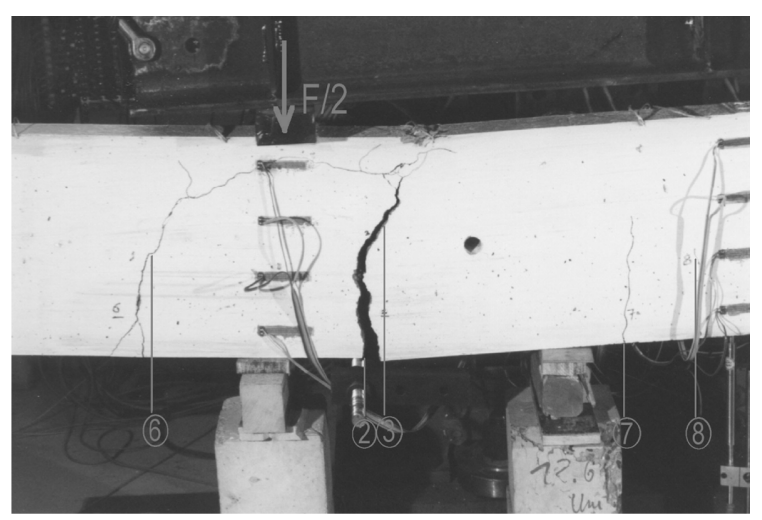

Fig. 4 Close-up around the crack that caused the failure for beam I.

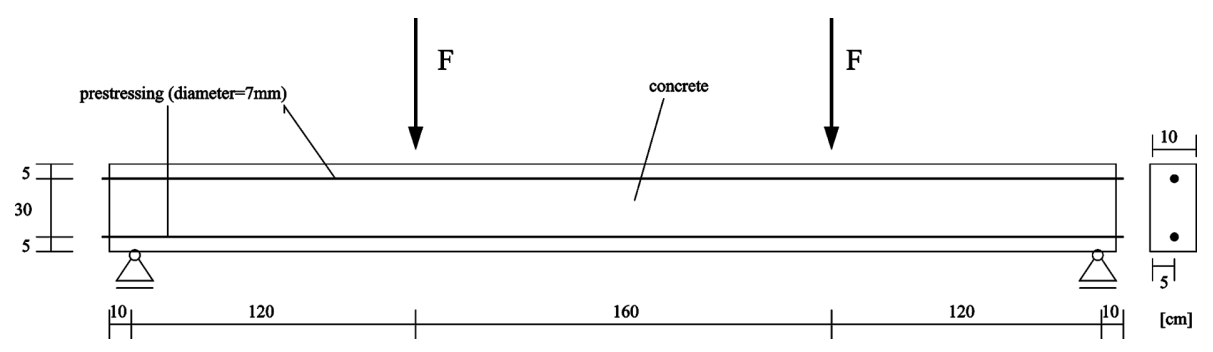

Fig. 3 Test set-up of beam I.

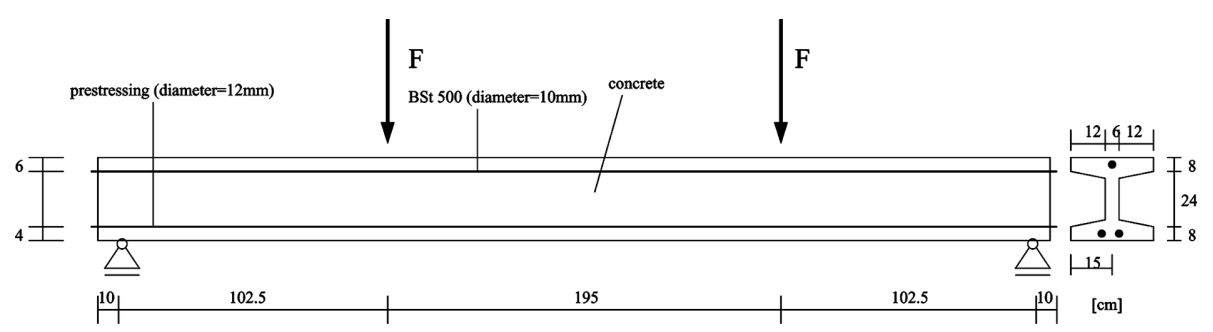

Fig. 5 Test set-up of beam II. 


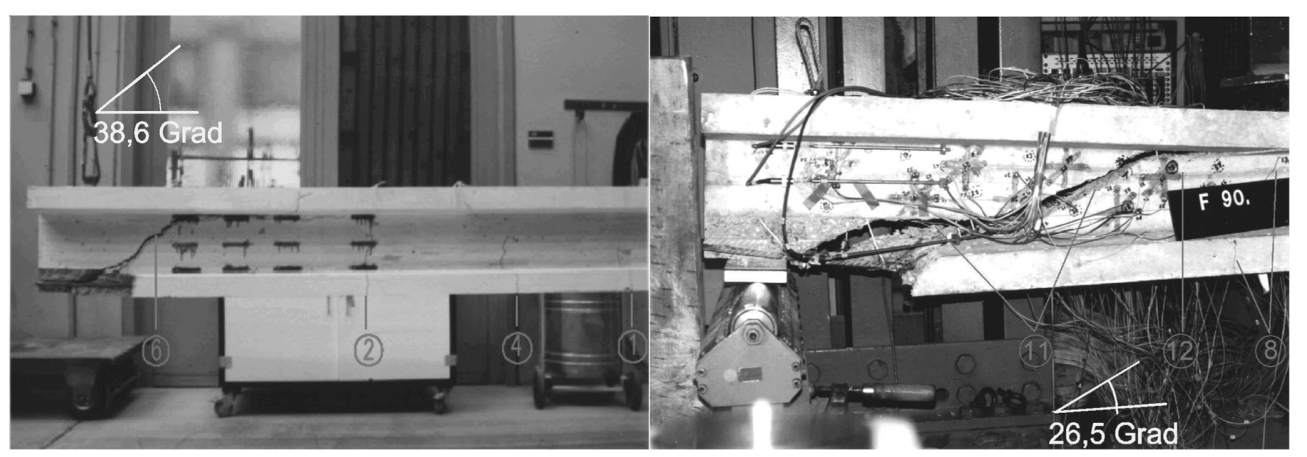

Fig. 6 Close-up around the crack that caused the failure for: LHS- beam II, RHS- beam III.

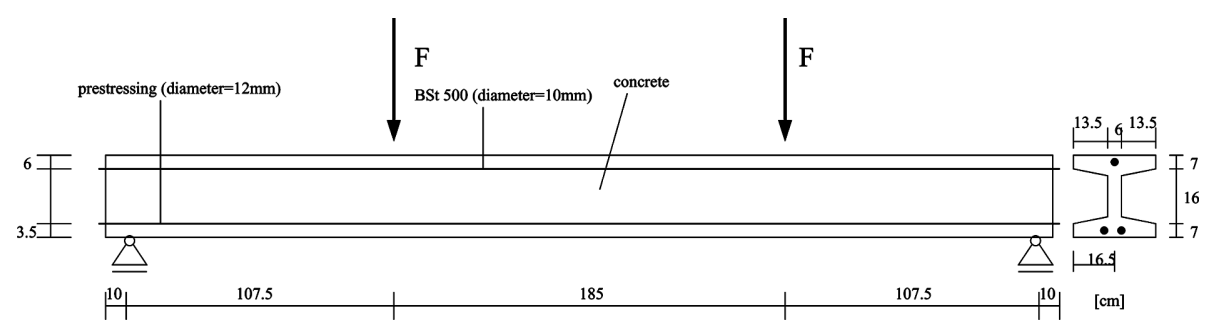

Fig. 7 Test set-up of beam III.

at the lower flange with a force of $68 \mathrm{kN}$ and the upper reinforcement (BSt 500, $10 \mathrm{~mm}$ ) was not prestressed. The same failure mechanism as for beam II was observed but the shear crack who caused the failure was farther from the support. More bending cracks appeared and the crack was flatter than in the second experiment. A picture of the beam after the experiment is shown in Fig. 6 (RHS). All beams were loaded by a displacement controlled approach.

We have used a bond model ${ }^{25}$ to take into account the interaction of the concrete and the reinforcement. Otherwise, the correct failure pattern cannot be reproduced. All concrete beams were discretized with particles while beam elements were used for the reinforcement. To solve the linearized systems of equations we employed the parallel direct solver Spooles based on overlapping domain decomposition. The computations were carried out on a parallel cluster using between 10 to 20 processors. To avoid an unrealistic symmetric crack pattern, we have varied the material strength in the specimen, meaning we multiplied the tensile strength with a small factor $1.2 \geq \alpha \geq 0.98$ obtained from a lognormal distribution around a mean value of 1 and a standard deviation of $2 \%$. We did not test the geometric correlation but it will influence the crack pattern; the global response (load-deflection curve) remains almost identical. Due to the large scatter of the crack patterns in the experiment, we find it reasonable not to study the geometric correlation, especially due to lack of information of the micro-structure.

The prestressing is modelled via a temperature loading case of the tension wires. In other words, the tension wire is shortened by cooling down. The strains are computed by $\varepsilon=\alpha_{T} \Delta T$ where $\alpha_{T}$ is the thermal expansion coefficient which is $1 \cdot 10^{-5} / \mathrm{C}$ for steel and $\Delta T$ is the temperature difference, which is negative in our case. The contraction of the tension wire transmits the prestressing forces in the concrete. In the experiments, the tension wires were first prestressed, then the beams were concreted and the prestressing forces were transmitted in the concrete after the desired com- pressive strength $(=45 \mathrm{MPa})$ was obtained.

Let us now focus on beam I: We have used an unstructured particle arrangement and varied the number of particles in the computation from 14,000 to 63,000 particles. In addition, we have used adaptivity in our computation to keep the computational cost low (twice lower compared to the coarsest discretization). We started the adaptive computations with approximately 8,000 particles and allowed 3 refinement steps. The adaptive refinement procedure and the error estimator is described in Rabczuk and Belytschko. ${ }^{24}$ Details about the constitutive model in the bulk, the cohesive model, the bond model and the coupling including all material data can be found in Rabczuk and Belytschko. ${ }^{25}$

Figure 8 shows the computed crack pattern at failure for beam I. The calculation reproduces the crack pattern well. Figure 9 shows the stresses for the tension wires at prestressing and a short time before the structure fails for the lower and upper wire, respectively. The prestressing obtained from the experiment is shown in figure 9 as well. At the beginning of the simulation (prestressing), a uniform distribution can be recognized. After $300 \mathrm{~mm}$ from the left support the stresses are completely transmitted in the steel. During loading, the stresses in the reinforcement increase, especially at locations where the concrete cracks and the reinforcement has to carry the load alone. Adjacent to the crack, the steel stresses in the reinforcement decrease.

The load displacement curve is shown in Fig. 10 for different computations. No mesh dependence occurs and the computational results agree with the experiment very well. A sudden drop in the load displacement curve at failure can be observed in the experiment and in the numerical simulation. The latter is caused due to a strain based failure criterion of the steel. Also the onset of the first cracking, when the stiffness of the structure is reduced due to the cracks, is detected by the simulation very well; it is the point where the load deflection curve starts to flatten. As can be seen, whenever a new crack is initiated a slight "jump" in the load deflection curve occurs caused by the decrease of the structural 
(a) Adaptive

(b) 14,000 particles

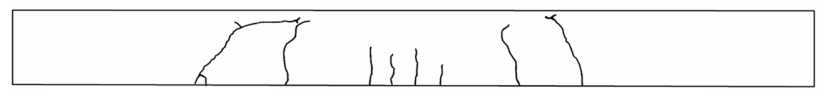

(c) Experiment

Fig. 8 Crack pattern of beam I: (a),(b) numerical simulation and (c) experiment.

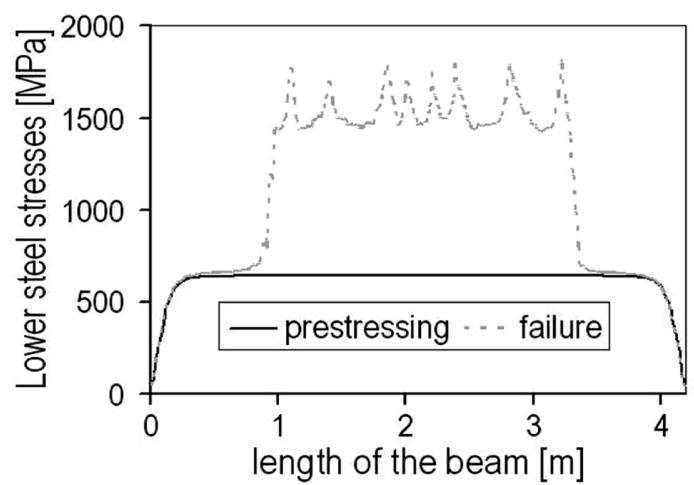

(a)

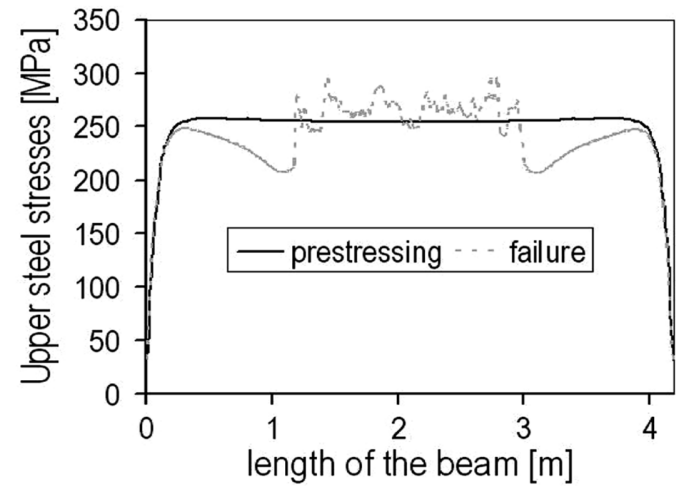

(b)

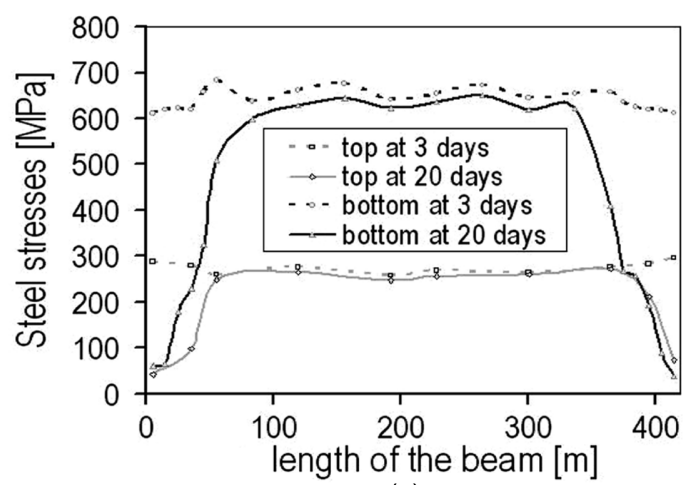

(c)

Fig. 9 Prestressing of the wires in (a),(b) the simulation and (c) the experiment; the experiment corresponds to the load case prestressing in the simulation.

stiffness.

For beam II, we also studied different discretizations, starting from 32,000 particles up to 120,000 particles. The adaptive com-

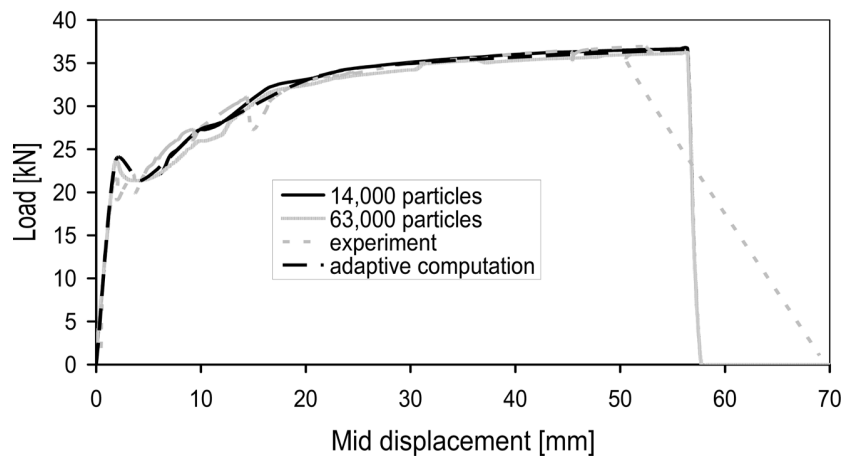

Fig. 10 Load deflection curve of beam I.

putation was performed with an initial number of particles of 13,000 particles and we allowed 2 refinement steps. Figure 11 shows the computed cracks at different load steps exemplarily for the coarsest particle discretization (32,000 particles). The shear crack in the experiment is closer to the support than in the computation probably due to some imperfections. However, the computation can predict the overall behaviour well, compare with Fig.12. The computed and experimental load mid-displacement curves for beam II are illustrated in Fig. 13 for different 'mesh' refinements and for the adaptive computation. The crack initiation is predicted by the simulation quite exact. Also the combined shear/anchorage failure is predicted well by the simulation. This is indicated by a sudden drop in the load deflection curve finally caused by the pullout of the lower tension wires, i.e. the bond length is shortened such that failure occurs. At first a shear crack is initiated at the transition between the lower flange and the web of the beam. This crack propagates towards the upper flange and simultaneously across the transition between the lower flange and the web in the direction of the closest support. The crack propagates further across the lower reinforcement and reduces the bond length at the end of the beam. A crack perpendicular to the crack that propagates at the transition of the web and the web separates two large

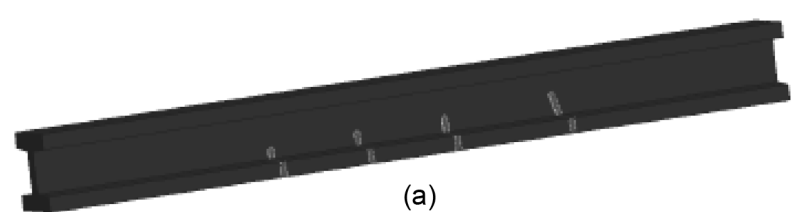

(a)

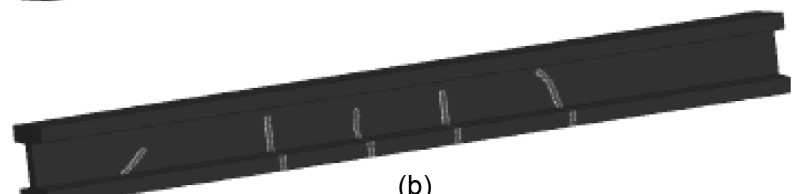

(b)

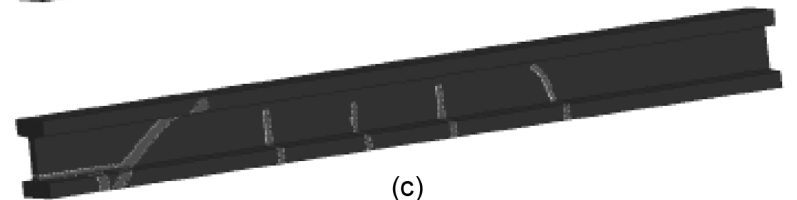

(c)

Fig. 11 Crack pattern of beam II obtained in the numerical simulation.

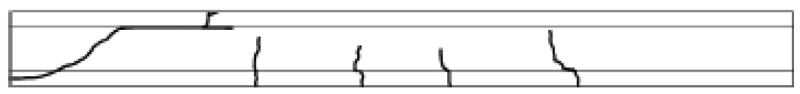

Fig. 12 Crack pattern of beam II in the experiment. 


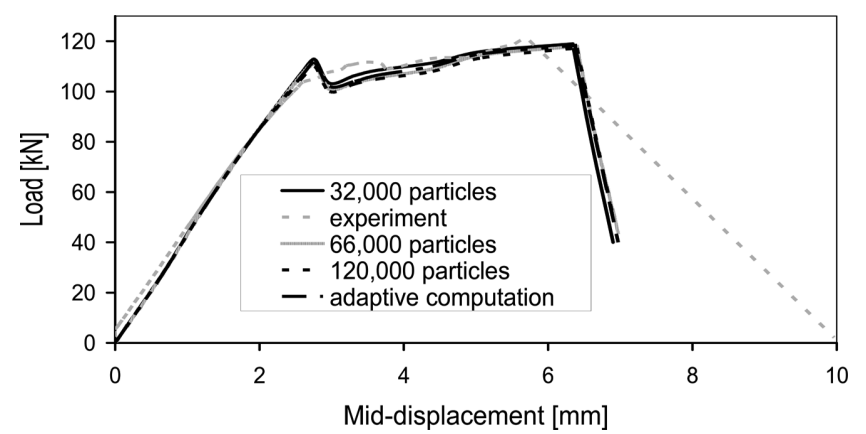

Fig. 13 Load deflection curve of beam II.

fragments from the beam. The sudden combined shear/anchorage failure is accompanied by a sudden drop in the load deflection curve, Fig. 13, and by a drop in the bond curve due to the reduction of the bond length, Fig. 14. As might be obvious, the failure occurred suddenly and the entire concrete breaks around the reinforcement. At that point the computation stopped to converge. We would like to mention that in an earlier paper, ${ }^{21}$ we were not able to capture the appropriate failure mechanism probably due to the two dimensional discretization.

The crack pattern for beam III at different load steps is illustrated in Fig. 15; Fig 15(c) shows the beam at failure. In contrast to beam II, more cracks occur and there are two shear cracks close to the support. This behaviour is captured by the simulation very well, compare with Fig. 16. Otherwise, the same observations as

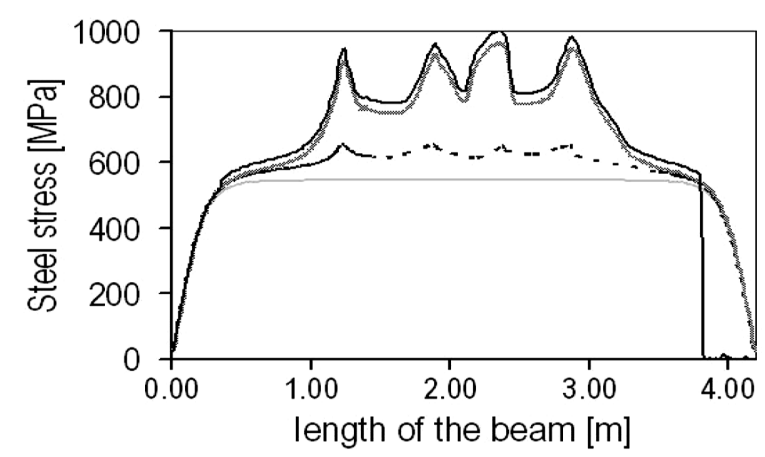

Fig. 14 Steel stresses at one lower tension wire at different load steps from the numerical simulation; beam II.
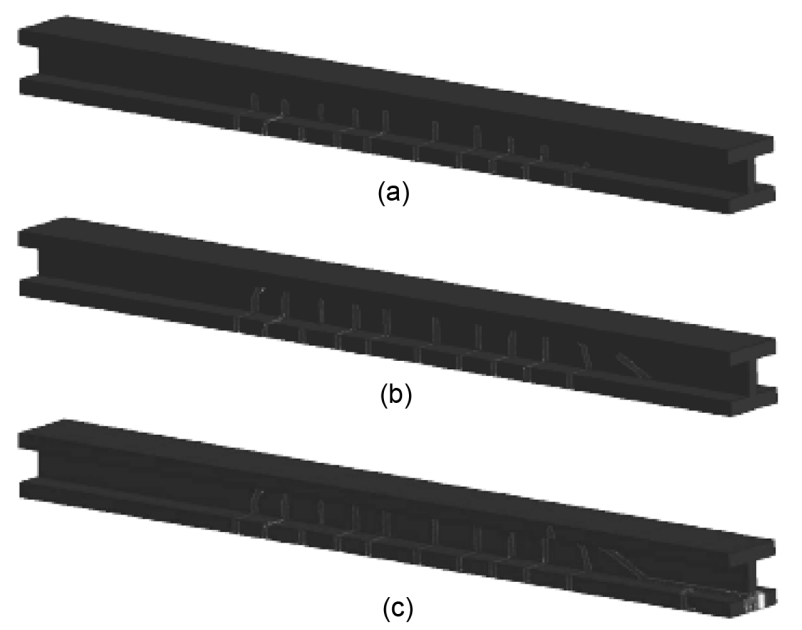

Fig. 15 Crack pattern of beam III at different load steps; numerical simulation.

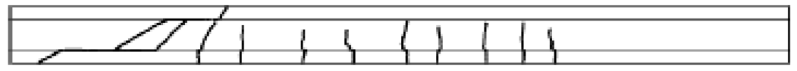

Fig. 16 Crack pattern of beam III in the experiment.

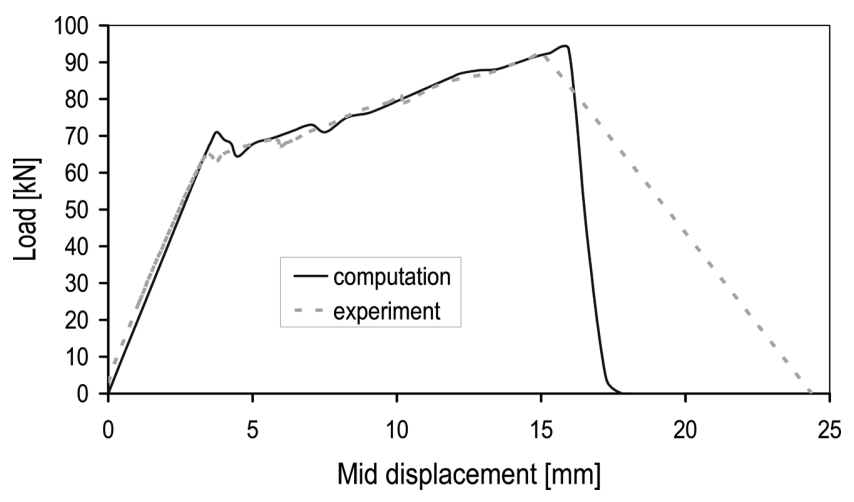

Fig. 17 Load deflection curve of beam III.

for beam II apply. The computed load deflection curve for beam III is shown in Fig. 17.

\section{Conclusions}

A new method for treating crack growth by particle methods has been proposed. In this method, the crack is treated as a set of cracked particles. Cohesive crack segments are located at the position of the cracked particles and the particle is split into two particles. The jump in the displacement field is obtained by the visibility method. Therefore, we proposed a simple and robust method to detect the connectivity of particles. The connectivity was removed across the crack surface. The major advantage of our method is that does not need a representation of the crack surface and hence, there is no need for algorithms that trace the crack paths.

The method is applied to prestressed concrete structures with multiple crack initiations and propagations. The numerical results were compared to experimental data and show excellent agreement. We were able to capture crack patterns, load deflection curves and different failure modes correctly.

\section{References}

1. F. Armero and K. Garikipati, "An analysis of Strong Discontinuities in Multiplicative Finite Strain Plasticity and Their Relation with the Numerical Simulation of Strain Localization in Solids," International Journal of Solids and Structures, Vol.33, No.20-22,:1996, pp. 2863 2885.

2. T. Belytschko and T. Black, "Elastic Crack Growth in Finite Elements with Minimal Remeshing," International Journal for Numerical Methods in Engineering, Vol. 45, No. 5. 1999, pp. 601 620.

3. T. Belytschko, J. Fish, and B. Englemann, "A Finite Element Method with Embedded Localization Zones," Computer Methods in Applied Mechanics and Engineering, Vol. 70, 1988, pp. 59 89.

4. T. Belytschko, M. Fleming, D. Organ, Y. Krongauz, and W.K. Liu, "Smoothing and Accelerated Computations in the Element Free Galerkin Method". Journal of Computational and Applied Mathematics, Vol 74, 1996, pp. 111 126.

5. T. Belytschko and Y.Y. Lu, "Element-Free Galerkin Methods 
for Static and Dynamic Fracture," International Journal of Solids and Structures, Vol. 32, 1995, pp. 2547 2570.

6. T. Belytschko, Y.Y. Lu, and L. Gu, "Crack Propagation by Element-Free Galerkin Methods," Engineering Fracture Mechanics, Vol. 51, No. 2, 1995, pp. 295 315.

7. T. Belytschko, N. Moes, S. Usui, and C. Parimi, "Arbitrary Discontinuities in Finite Elements," International Journal for Numerical Methods in Engineering, Vol 50, No. 4, pp. 993 1013.

8. T. Belytschko, D. Organ, and M. Tabbara, "Numerical Simulations of Mixed Mode Dynamic Fracture in Concrete Using Element-free Galerkin Methods," ICES Conference Proceedings, 1995.

9. T. Belytschko and M. Tabbara, "Dynamic Fracture Using Element-free Galerkin Methods," International Journal for Numerical Methods in Engineering, Vol 39, No. 6, 1996, pp. 923 938.

10. G. T. Camacho and M. Ortiz, "Computational Modeling of Impact Damage in Brittle Materials," International Journal of Solids and Structures, Vol. 33, 1996, pp.2899 2938.

11. C. Daux, N. Moes, J. Dolbow, N. Sukumar, and T. Belytschko, "Arbitrary Branched and Intersection Cracks with the Extended Finite Element Method," International Journal for Numerical Methods in Engineering, Vol. 48, 2000, pp. 1731 1760.

12. N. Hermann. Experimentelle Erfassung des Betonverhaltens unter Schockwellen. $\mathrm{PhD}$ thesis, Institut fuer Massivbau und Baustofftechnologie, Universitaet Karlsruhe, 2002.

13. P. Krysl and T. Belytschko, "The Element Free Galerkin Method for Dynamic Propagation of Arbitrary 3-D Cracks," International Journal for Numerical Methods in Engineering, Vol. 44, No. 6, 1999, pp. 767 800.

14. J. Lemaitre, "Evaluation of Dissipation and Damage in Metal Submitted to Dynamic Loading," Proceedings ICM 1, 1971.

15. N. Moes, J. Dolbow, and T. Belytschko, "A Finite Element Method for Crack Growth without Remeshing," International Journal for Numerical Methods in Engineering, Vol.46, No. 1, 1999, pp. 133 150.

16. J. Oliver, "Modelling Strong Discontinuities in Solid Mechanics via Strain Softening Constituitive Equations, Part 1: Fundamentals. Part 2: Numerical Simulation," International Journal for Numerical Methods in Engineering, Vol. 39, 1996, pp. 3575 3624.

17. J. Oliver, M. Cervera, and O. Manzoli, "Strong Discontinuities and Continuum Plasticity Models: the Strong Discontinuity Approach," International Journal of Plasticity, Vol 15, 1999, pp. 319 351.

18. J. Oliver, A.E. Huespe, and P.J. Snchez, "A Comparative Study on Finite Elements for Capturing Strong Discontinuities: EFem vs. x-Fem," Computer Methods in Applied Mechanics and Engineering, in press, 2006.

19. D. Organ, M. Fleming, T. Terry, and T. Belytschko, "Continuous Meshless Approximations for Nonconvex Bodies by Diffraction and Transparency," Computational Mechanics, Vol 18, 1996, pp. $225 \sim 235$.

20. M. Ortiz, Y. Leroy, and A. Needleman, "Finite Element Method for Localized Failure Analysis," Computer Methods in Applied Mechanics and Engineering, Vol. 61, No. 2, 1987, pp. 189 214.

21. T. Rabczuk, J. Akkermann, and J. Eibl, “A Numerical Model for Reinforced Concrete Structures," International Journal of Solids and Structures, 5-6, 2005, pp. 1327 1354, 42.

22. T. Rabczuk, P.M.A. Areias, and T. Belytschko, "A Meshfree Thin Shell for Large Deformation, Finite Strain and Arbitrary Evolving Cracks," International Journal for Numerical Methods in Engineering, Vol. 72, No. 5, 2007, pp. 524 548.

23. T. Rabczuk and T. Belytschko, "Cracking Particles: A Simplified Meshfree Method for Arbitrary Evolving Cracks," International Journal for Numerical Methods in Engineering, Vol. 61, No. 13, 2004, pp. 2316 2343.

24. T. Rabczuk and T. Belytschko, "Adaptivity for Structured Meshfree Particle Methods in 2D and 3D," International Journal for Numerical Methods in Engineering, Vol. 63, No. 11, 2005, pp. 1559 1582.

25. T. Rabczuk and T. Belytschko, "Application of Meshfree Particle Methods to Static Fracture of Reinforced Concrete Structures," International Journal of Fracture, 137(1-4), 2006, pp. 19 49.

26. T. Rabczuk and T. Belytschko, "A Three Dimensional Large Deformation Meshfree Method for Arbitrary Evolving Cracks," Computer Methods in Applied Mechanics and Engineering, 196 (29-30), 2007, pp. 2777 2799.

27. T. Rabczuk, T. Belytschko, and S.P. Xiao, "Stable Particle Methods Based on Lagrangian Kernels," Computer Methods in Applied Mechanics and Engineering, 193:1035 1063, 2004.

28. J. J. C. Remmers, R. de Borst, and A. Needleman, "A Cohesive Segments Method for the Simulation of Crack Growth," Computational Mechanics, Vol. 31, 2003, pp. 69 77.

29. X.-P. Xu and A. Needleman, "Void Nucleation by Inclusion Debonding in a Crystal Matrix," Modell. Simul. Mater. Sci. Engng., Vol. 1, 1993, pp. 111 132.

30. X.-P. Xu and A. Needleman, "Numerical Simulations of Fast Crack Growth in Brittle Solids," Journal of the Mechanics and Physics of Solids, Vol. 42, 1994, pp. 1397 1434.

31. F. Zhou and J.F. Molinari, "Dynamic Crack Propagation with Cohesive Elements: a Methodolgy to Address Mesh Dependence," International Journal for Numerical Methods in Engineering, Vol. 59, No. 1, 2004, pp. 1 24.

32. S. Bordas and B. Moran, "Enriched Finite Elements and Level Sets for Damage Tolerance Assessment of Complex Structures," Engineering Fracture Mechanics, Vol 73, 2006, pp. 1176 1201.

33. R. Duddu and S. Bordas and B. Moran, "A Combined Extended Finite Element and Level set Method for Biofilm Growth," International Journal for Numerical Methods in Engineering, Vol. 74, No. 5, 2008, pp. 848 870.

34. S. Bordas and V.P. Nguyen and C. Dunant and H. NguyenDang and A. Guidoum, "An Extended Finite Element Library," International Journal for Numerical Methods in Engineering, Vol. 71, No. 6, 2007, pp. 703 732.

35. S. Bordas and J.G. Conley and B. Moran and J. Gray and E. Nichols, "A Simulation-Based Design Paradigm for Complex Cast Components," Engineering with Computers, Vol. 23, No. 1, 2007, pp. $25 \sim 37$.

36. L. Stempniewski and J. Eibl and T. Rabczuk, Der Endbereich von im Werk vorgespannten Fertigteiltraegern-Hohlplatten, Final report, University of Karlsruhe, Institut fuer Massivbau und Baustofftechnologie, 2001. 